\title{
Clinical, Virologic, and Epidemiologic Characteristics of Dengue Outbreak, Dar es Salaam, Tanzania, 2014
}

\author{
Francesco Vairo, ${ }^{1}$ Leonard E.G. Mboera, ${ }^{1}$ \\ Pasquale De Nardo, Ndekya M. Oriyo, \\ Silvia Meschi, Susan F. Rumisha, \\ Francesca Colavita, Athanas Mhina, \\ Fabrizio Carletti, Elibariki Mwakapeje, \\ Maria Rosaria Capobianchi, Concetta Castilletti, \\ Antonino Di Caro, Emanuele Nicastri, \\ Mwelecele N. Malecela, ${ }^{2}$ Giuseppe Ippolito ${ }^{2}$
}

We investigated a dengue outbreak in Dar es Salaam, Tanzania, in 2014, that was caused by dengue virus (DENV) serotype 2. DENV infection was present in 101 (20.9\%) of 483 patients. Patient age and location of residence were associated with infection. Seven (4.0\%) of 176 patients were co-infected with malaria and DENV.

$\mathrm{D}$ ata are scarce on seroprevalence of dengue virus (DENV) in Tanzania. Cross-sectional studies conducted during 2007-2014 indicated that DENV seroprevalence ranged from $<2 \%$ to $>50 \%$, depending on geographic area and epidemiologic characteristics of patients (1-6). Aedes aegypti mosquitoes, the main vector of DENV, are present throughout Tanzania (7), and the clinical course of DENV infection is greatly affected by previous exposure to different DENV serotypes (8). Investigation of DENV outbreaks might serve to define circulation of different serotypes and the best strategy to manage future outbreaks.

In 2014, a large dengue outbreak occurred in Dar es Salaam, Tanzania (8). We report the main findings of a study conducted there during the outbreak.

\section{The Study}

Ethical approval for the study was obtained from the National Health Research Ethics Sub-Committee of Tanzania (protocol no. NIMR/HQ/R.8a/Vol. IX/I733). Informed consent was obtained from all participants.

Author affiliations: National Institute for Infectious Diseases

L. Spallanzani, Rome, Italy (F. Vairo, P. De Nardo, S. Meschi,

F. Colavita, F. Carletti, M.R. Capobianchi, C. Castilletti, A. Di Caro,

E. Nicastri, G. Ippolito); National Institute for Medical Research,

Dar es Salaam, Tanzania (L.E.G. Mboera, N.M. Oriyo, S.F.

Rumisha, A. Mhina, M.N. Malecela); Ministry of Health and Social Welfare, Dar es Salaam (E. Mwakapeje)

DOI: http://dx.doi.org/10.3201/eid2205.151462
The study involved the 3 districts of Dar es Salaam (Kinondoni, Ilala, and Temeke (Figure 1, panel A). All consecutive patients with fever (temperature $>37.5^{\circ} \mathrm{C}$ ) for $<7$ days who came to the outpatient department of 1 of 7 selected health facilities were tested for malaria by using a malaria rapid diagnostic test (mRDT) (SD Bioline Malaria Ag. Pf/Pan Test, Bioline, Gewerbestrasse, Switzerland) and for dengue by using a DENV rapid diagnostic test (dRDT) (SD Bioline Dengue Duo; Standard Diagnostics, Inc., Gyeonggi-do, South Korea).

DENV infection was defined as a positive result for DENV nonstructural protein 1 (NS1) or IgM against DENV detected by dRDT. Past DENV infection was defined as IgG against DENV alone detected by dRDT. Malaria infection was defined as a positive result by mRDT. Warning signs and severe dengue were defined according to guidelines of the World Health Organization (9). A structured interview was used to collect demographic, environmental, and clinical data. Molecular analyses were conducted at the National Institute for Infectious Diseases L. Spallanzani (Rome, Italy).

Virus RNA was extracted and 2 real-time reverse transcription PCRs (RT-PCRs), one specific for DENV serotypes 1, 2, and 3 and one for DENV serotype 4, were performed on dRDT-positive serum samples as described $(10,11)$. For identification of DENV serotypes, a fragment spanning the E-NS1 gene junction was amplified as described (12). A complete envelope (E) gene sequence was obtained by using a One-Step RT-PCR Kit (QIAGEN, Hilden, Germany) and primers (primer sequences available on request). Sanger sequencing and phylogenetic analysis based on the nucleotide sequence of the E-NS1 region and a complete $\mathrm{E}$ gene sequence (Figure 2) were performed. A multiple logistic regression model with a backward procedure was used to determine a cutoff level of $\mathrm{p}=0.10$.

A total of 483 of 491 patients who came to health facilities and matched inclusion criteria were enrolled in the study. Among enrolled patients, 101 (20.9\%) were positive for DENV infection, and $9(1.9 \%)$ were positive for past DENV infection. Incidence of DENV infections peaked during mid-June and decreased toward the end of the month (Figure 1, panel B).

${ }^{1}$ These first authors contributed equally to this article.

${ }^{2}$ These senior authors contributed equally to this article. 


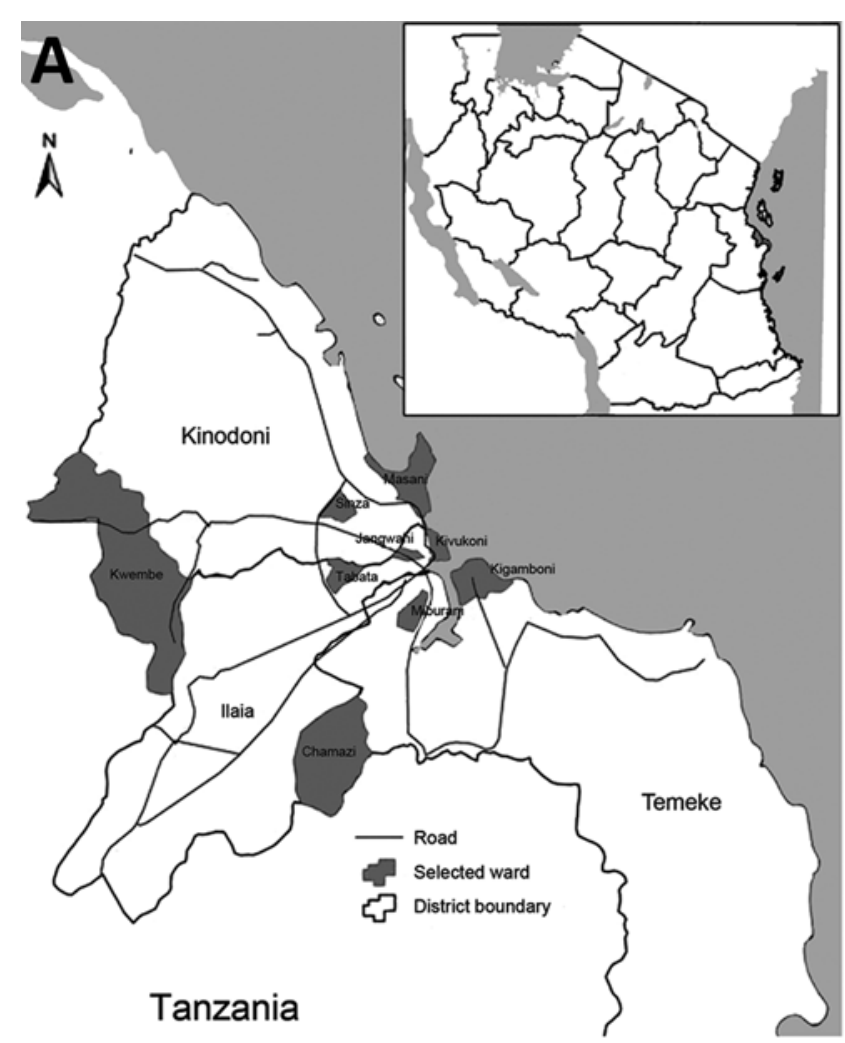

Univariate analysis showed that current DENV infection was associated with patient age $(p<0.001)$, location of residence $(p=0.007)$, and employment status $(p<0.001)$ (Table). Multivariate analysis showed that only age and location of residence were independently associated with current DENV infection. The risk for infection for patients $>15$ years of age was twice that for patients $\leq 15$ years of age (odds ratio $2.54,95 \%$ CI $1.10-5.87 ; p=0.029$ ). Patients who lived in Kinondoni were twice as likely to have a DENV infection than patients who lived in Temeke (odds ratio 2.83, 95\% CI 1.44-5.56; $\mathrm{p}=0.002$ ).

Only joint and muscle pain were associated with DENV infection $(\mathrm{p}<0.001)$. Warning signs were more frequent in patients with DENV infection (42/101, 41.6\%; $\mathrm{p}=0.006$ ). Three patients met criteria for severe dengue. Patients with DENV infection were more likely to be hospitalized $(\mathrm{p}<0.001)$ and to have used antimalarial drugs ( $\mathrm{p}$ $=0.025)$. A total of $176(34.6 \%)$ patients had a positive result for malaria by mRDT. Of these patients, 7 (4.0\%) were co-infected with DENV (positive result for NS1), and $14(8.0 \%)$ had a recent DENV-malaria coinfection (IgM or IgM/IgG positive). Sixty-two patients with DENV infection had positive results by PCR.

Sequence analysis of NS1-E junction gene fragments of DENV was performed for 8 randomly selected RT-PCRpositive samples (GenBank accession nos. KT288895KT288902). Phylogenetic analysis of all sequences

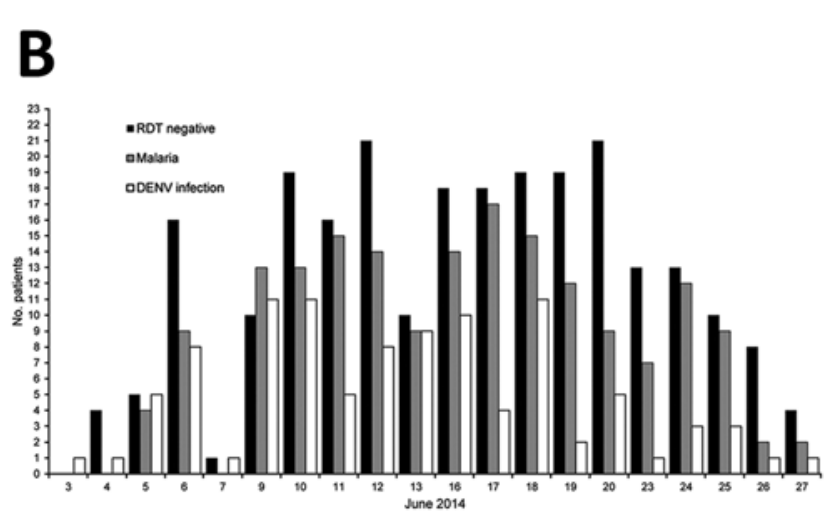

Figure 1. Geographic and clinical details of dengue outbreak, Dar es Salaam, Tanzania, 2014. A) Location of 3 districts investigated. *Districts with no health facility available during the study. Outpatient departments were not open on weekends. Inset indicates location of Dar el Salaam in Tanzania (black). B) No. cases of dengue virus (DENV) infection and malaria and rapid diagnostic test (RDT) results during the outbreak.

grouped them in a monophyletic cluster in the DENV serotype 2 cosmopolitan genotype. Further sequencing of the complete E gene was performed for 1 isolate (GenBank accession no. KT288902). Clustering with the DENV serotype 2 cosmopolitan genotype was confirmed (Figure 2 ). This analysis showed similarity of DENV in Tanzania with those from Asia isolated since 2001 and a strong phylogenetic relationship (99.8\% identity) with a DENV variant isolated in Guangzhou, China, in 2013 (GenBank accession no. KJ807797).

\section{Conclusions}

We report DENV serotype 2 (Cosmopolitan genotype) as the causative agent of the dengue outbreak in Dar es Salaam in 2014. Sequence analysis showed that this virus from Tanzania had a strong phylogenetic relationship with virus strains from China, India, East Timor, and Singapore. These results indicate that the virus could have been recently introduced into Tanzania by travelers from Asia. This hypothesis is supported by the phylogenetic relationship with sequences obtained from isolates in Asia since 2001 and by reports of DENV-3 detection in the previous years in Zanzibar $(4,5)$.

The incidence of DENV infection in Dar es Salaam $(20.9 \%)$ is higher than incidences reported in recent studies of household participants in Angola (9\% of recent infections) (13) and Kenya (13\% of recent/current infections). This discordance might be related to different 


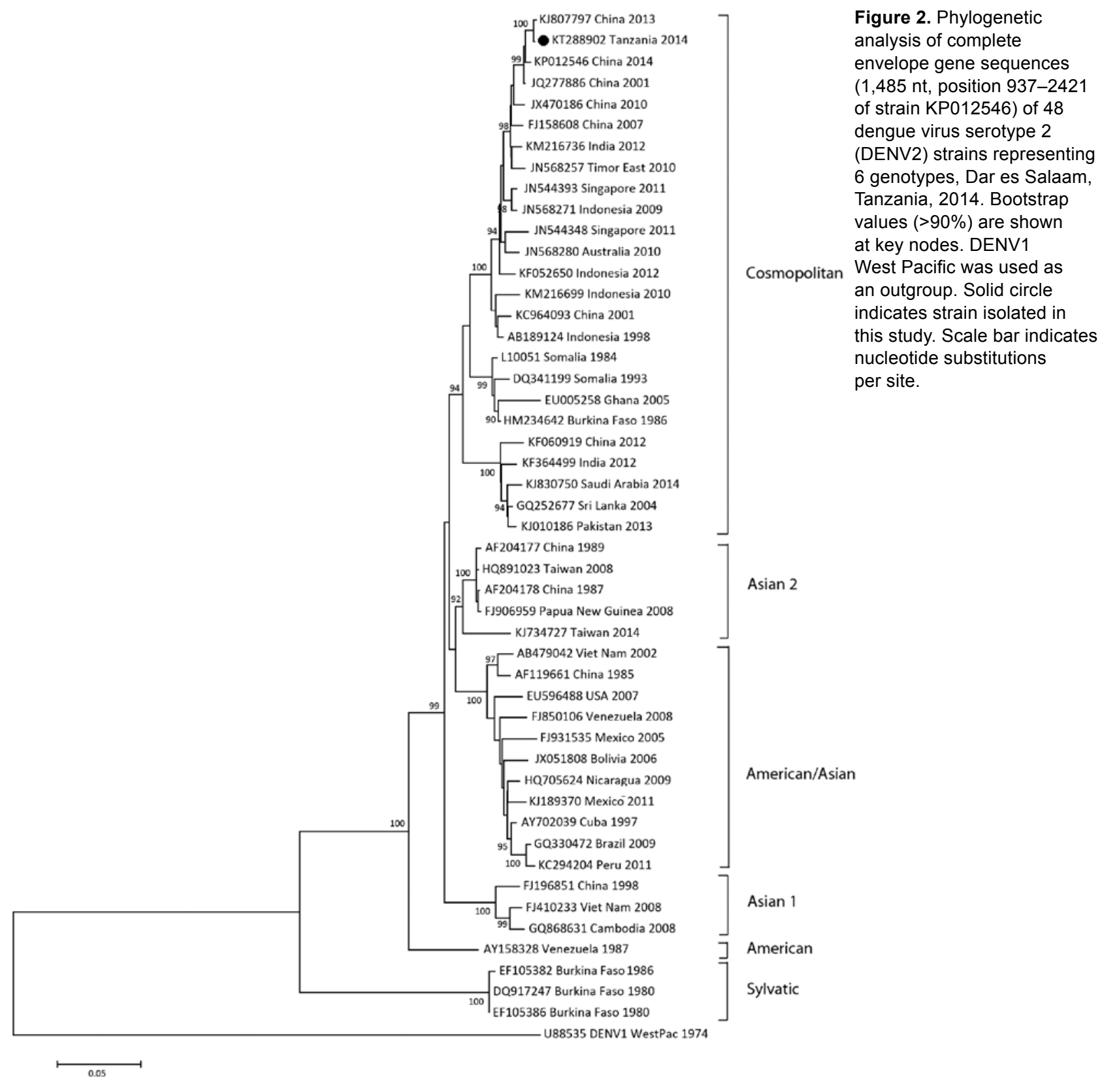

designs of these 2 studies. Our study was conducted with febrile patients attending health facilities and not in households. Moreover, the dRDT used in our study has a lower specificity than that of tests used in Angola and Kenya.

The higher incidence of DENV infection among elderly patients is not consistent with recent data for other disease-endemic regions (13) and might be related to possible recent introduction of the DENV serotype 2 in the area. The higher incidence reported in Kinondoni District could be related to the urban nature of the district.
As reported in Africa $(13,14)$, no environmental or behavioral factors were associated with DENV infection. Malaria and DENV co-infection was present in $4 \%$ of patients, a find similar to that in a recent study in Ghana, where possible co-infection in was reported in $3.2 \%$ of children (15). As reported in Kenya (14), the high use rate (33.3\%) for antimalaria treatment in patients with DENV infection during the current outbreak suggests a high level of underrecognition of dengue.

External validity of our results should be evaluated according to potential limitations of the study. First, the study used passive recruitment, which might have 
Table. Characteristics of 483 patients tested for DENV infection during dengue outbreak, Dar es Salaam, Tanzania, 2014*

\begin{tabular}{|c|c|c|c|c|}
\hline Characteristic & Current dengue infection, $n=101$ & No dengue, $\mathrm{n}=382$ & Total, $n=483$ & $p$ value \\
\hline Sex & & & & $0.067 \dagger$ \\
\hline M & $65(64.4)$ & $207(54.2)$ & $272(56.3)$ & NA \\
\hline $\mathrm{F}$ & $36(35.6)$ & $175(45.8)$ & $208(43.7)$ & NA \\
\hline Median age, y (IQR) & $28(21-37)$ & $24(13-35)$ & $25(14-36)$ & $<0.001 \ddagger$ \\
\hline Age, $y, n=481$ & & & & $<0.001 \dagger$ \\
\hline$\leq 15$ & $12(11.9)$ & $117(30.8)$ & $129(26.8)$ & NA \\
\hline$>15$ & $89(88.1)$ & $263(69.2)$ & $352(73.2)$ & NA \\
\hline District & & & & $0.007 \dagger$ \\
\hline Temeke & $18(17.8)$ & $123(32.2)$ & $141(29.2)$ & NA \\
\hline Ilala & $34(33.7)$ & $128(33.5)$ & $162(33.5)$ & NA \\
\hline Kinondoni & $49(48.5)$ & $131(34.3)$ & $180(37.3)$ & NA \\
\hline Employed, $\mathrm{n}=455$ & $66(69.5)$ & $169(46.9)$ & $235(51.7)$ & $<0.001 \dagger$ \\
\hline Water storage, $n=476$ & $43(43.9)$ & $185(48.9)$ & $228(47.9)$ & $0.371 \dagger$ \\
\hline Resting water, $n=481$ & $48(48.5)$ & $179(46.9)$ & $227(47.2)$ & $0.773 \dagger$ \\
\hline Persons in household, $\mathrm{n}=474$ & & & & $0.284 \dagger$ \\
\hline $1-3$ & $33(33.3)$ & $118(31.5)$ & $151(31.9)$ & NA \\
\hline $4-6$ & $41(41.4)$ & $185(49.3)$ & $226(47.7)$ & NA \\
\hline$\geq 7$ & $25(19.2)$ & $72(19.2)$ & $97(20.5)$ & NA \\
\hline Bed net use, $n=479$ & $75(75)$ & $313(82.6)$ & $388(81)$ & $0.085 \dagger$ \\
\hline Insecticide spraying in home, $n=479$ & $20(20)$ & $87(21)$ & $107(22.3)$ & $0.528 \dagger$ \\
\hline
\end{tabular}

resulted in potential selection bias (only sicker patients who came to selected health facilities were analyzed). Second, the study had virtually no follow-up, which precluded any inferences about clinical outcomes of patients with severe dengue.

Despite these limitations, our study provides useful information on an underreported disease and on the molecular epidemiology of largest dengue outbreak reported in Tanzania. Results of our study can improve awareness of healthcare providers and demonstrate the feasibility of interventions to enhance diagnostic testing capabilities and specific surveillance systems. Resurgence of dengue in Tanzania should prompt implementation of population-based studies on differential diagnosis of acute febrile illness and surveillance systems based on syndromic approaches.

\section{Acknowledgments}

We thank the commissioners and medical officers of Ilala,

Temeke, and Kinondoni Districts for their cooperation; the medical officers in charge of health facilities for providing technical and logistic support; the director general of the National Institute for Medical Research for providing permission to publish this article; the World Health Organization (Tanzania Country Office) for providing dengue rapid diagnostic tests; the National Health Laboratory Quality Assurance and Training Center for providing technical support for sample separation and storage; Carolyn Riwa, Serena Quartu, Paola Zaccaro, Maria Kelly, Solomon Mwakasungula, and Alfred Mwenda for processing and storing samples; and Alessandro Pandolfo, Onesmo Kateule, and Neema Camara for providing support during recruitment of patients and collection of samples at health facilities.
This study was supported by the National Institute for Medical Research, Tanzania; Ministry of Health and Social Welfare, Tanzania; Ministry of Health, Ricerca Corrente, Italy; and the Directorate General of the Italian Cooperation, Italian Ministry of Foreign Affairs (project AID9562).

Dr. Vairo is an infectious disease specialist and epidemiologist in the Epidemiology Department, National Institute for Infectious Diseases L. Spallanzani, Rome, Italy. His main research interests are the epidemiology of emerging infectious diseases and outbreak preparedness and response.

\section{References}

1. Vairo F, Nicastri E, Meschi S, Schepisi MS, Paglia MG, Bevilacqua N, et al. Seroprevalence of dengue infection: a cross-sectional survey in mainland Tanzania and on Pemba Island, Zanzibar. Int J Infect Dis. 2012;16:e44-6. http://dx.doi.org/ 10.1016/j.ijid.2011.09.018

2. Hertz JT, Munishi OM, Ooi EE, Howe S, Lim WY, Chow A, et al. Chikungunya and dengue fever among hospitalized febrile patients in northern Tanzania. Am J Trop Med Hyg. 2012;86:171-7. http://dx.doi.org/10.4269/ajtmh.2012.11-0393

3. Vairo F, Nicastri E, Yussuf SM, Cannas A, Meschi S, Mahmoud MA, et al. IgG against dengue virus in healthy blood donors, Zanzibar, Tanzania. Emerg Infect Dis. 2014;20:465-8. http://dx.doi.org/10.3201/eid2003.130150

4. Moi ML, Takasaki T, Kotaki A, Tajima S, Lim CK, Sakamoto M, et al. Importation of dengue virus type 3 to Japan from Tanzania and Côte d'Ivoire. Emerg Infect Dis. 2010;16:1770-2. http://dx.doi.org/10.3201/eid1611.101061

5. Ippolito G. Dengue: Italy ex Tanzania. ProMED. July 9, 2010 [cited 2016 Jan 12]. http://www.promedmail.org, archive no 20100709.2304.

6. Chipwaza B, Mugasa JP, Selemani M, Amuri M, Mosha F, Ngatunga SD, et al. Dengue and Chikungunya fever among viral diseases in outpatient febrile children in Kilosa district hospital, Tanzania. PLoS Negl Trop Dis. 2014;8:e3335. http://dx.doi.org/10.1371/journal.pntd.0003335 
7. Mweya CN, Kimera SI, Mellau LS, Mboera LE. Inter-epidemic abundance and distribution of potential mosquito vectors for Rift Valley fever virus in Ngorongoro district, Tanzania. Glob Health Action. 2015;8:25929.

8. World Health Organization. Dengue outbreak in the United Republic of Tanzania (situation as of 30 May 2014) [cited 2016 Jan 12]. http://www.afro.who.int/pt/grupos-organicos-e-programas/ ddc/alerta-e-resposta-epidemias-e-pandemias/4155-dengue-outbreak-in-the-united-republic-of-tanzania-30-may-2014.html

9. World Health Organization. Dengue. Gguidelines for diagnosis, treatment, prevention and control, November 2009 [cited 2016 Jan 12]. http://whqlibdoc.who.int/publications/2009/ 9789241547871_eng.pdf

10. Gurukumar KR, Priyadarshini D, Patil JA, Bhagat A, Singh A, Shah PS, et al. Development of real time PCR for detection and quantitation of dengue viruses. Virol J. 2009;6:10. http://dx.doi.org/ 10.1186/1743-422X-6-10

11. Leparc-Goffart I, Baragatti M, Temmam S, Tuiskunen A, Moureau G, Charrel R, et al. Development and validation of real-time one-step reverse transcription-PCR for the detection and typing of dengue viruses. J Clin Virol. 2009;45:61-6. http://dx.doi.org/10.1016/j.jcv.2009.02.010
12. Domingo C, Palacios G, Niedrig M, Cabrerizo M, Jabado O, Reyes N, et al. A new tool for the diagnosis and molecular surveillance of dengue infections in clinical samples. Dengue Bulletin. 2004;28:87-95.

13. Sharp TM, Moreira R, Soares MJ, Miguel da Costa L, Mann J, DeLorey M, et al. Underrecognition of dengue during 2013 epidemic in Luanda, Angola. Emerg Infect Dis. 2015;21:1311-6. http://dx.doi.org/10.3201/eid2108.150368

14. Ellis EM, Neatherlin JC, Delorey M, Ochieng M, Mohamed AH, Mogeni DO, et al. A household serosurvey to estimate the magnitude of a dengue outbreak in Mombasa, Kenya, 2013. PLoS Negl Trop Dis. 2015;9:e0003733. http://dx.doi.org/10.1371/ journal.pntd.0003733

15. Stoler J, Delimini RK, Bonney JH, Oduro AR, Owusu-Agyei S, Fobil JN, et al. Evidence of recent dengue exposure among malaria parasite-positive children in three urban centers in Ghana. Am J Trop Med Hyg. 2015;92:497-500. http://dx.doi.org/10.4269/ ajtmh.14-0678

Address for correspondence: Francesco Vairo, National Institute for Infectious Diseases L. Spallanzani, Via Portuense 292, 00146, Rome, Italy; email: francesco.vairo@inmi.it

\section{July 2015: Malaria Including:}

- Disseminated Infections with Talaromyces marneffei in Non-AIDS Patients Given Monoclonal Antibodies against CD2O and Kinase Inhibitors

- Macacine Herpesvirus 1 in Long-Tailed Macaques, Malaysia, 2009-2011

- Malaria Prevalence among Young Infants in Different Transmission Settings, Africa

- Transdermal Diagnosis of Malaria Using Vapor Nanobubbles

- Lack of Transmission among Close Contacts of Patient with Case of Middle East Respiratory Syndrome Imported into the United States, 2014

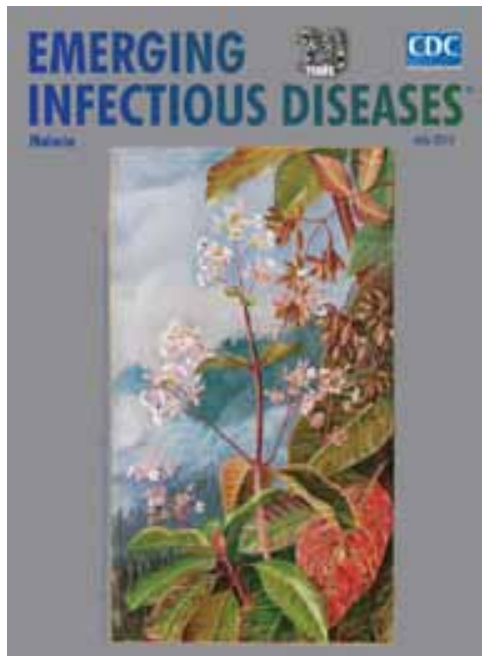

- Monitoring of Ebola Virus Makona Evolution through Establishment of Advanced Genomic Capability in Liberia

- Parechovirus Genotype 3 Outbreak among Infants, New South Wales, Australia, 2013-2014

- MERS-CoV in Upper Respiratory Tract and Lungs of Dromedary Camels, Saudi Arabia, 2013-2014

- Assessment of Arbovirus Surveillance 13 Years after Introduction of West Nile Virus, United States

- Results from the National Legionella Outbreak Detection Program, the Netherlands, 2002-2012

http://wwwnc.cdc.gov/eid/articles/issue/21/07/table-of-contents 